\title{
Antibody Response 3 Months after 2 Doses of BNT162b2 mRNA COVID-19 Vaccine in Residents of Long-Term Care Facilities
}

\author{
Roberta Causa ${ }^{a, b}$ Diego Almagro-Nievas ${ }^{a} \quad$ Mario Rivera-Izquierdo ${ }^{\text {a, c, d, e }}$ \\ Nicolás Benítez-Muñoz ${ }^{a}$ Begoña López-Hernández ${ }^{a}$ Fernando García-Garcíad, $f$ \\ Marta Alvarez-Estévez ${ }^{d, f} \quad$ Maria de la O. Soto-Pérez ${ }^{a} \quad$ Clara Bermúdez-Tamayo ${ }^{\text {b, } g}$ \\ aDistrito Sanitario Granada-Metropolitano, Granada, Spain; bescuela Andaluza de Salud Pública, Granada, Spain; \\ 'Service of Preventive Medicine and Public Health, Hospital Universitario San Cecilio, Granada, Spain; ${ }^{d}$ Instituto de \\ Investigación Biosanitaria de Granada (ibs, GRANADA), Granada, Spain; 'Department of Preventive Medicine and Public \\ Health, University of Granada, Granada, Spain; 'Service of Microbiology, Hospital Universitario San Cecilio, Granada, \\ Spain; ' Centro de Investigación Biomédica en Red de Epidemiología y Salud Pública (CIBERESP), Madrid, Spain
}

\section{Keywords}

COVID-19 · Antibody response $\cdot$ Long-term care facilities · BNT162b2 Immunosenescence

\begin{abstract}
Background: Older adults living in long-term care facilities (LTCFs) are at increased risk for severe outcomes from COVID-19 and were identified as a priority group in COVID-19 vaccination strategies. Emerging evidence suggests vaccine effectiveness in LTCF populations, but data about median and long-term durability of immune response after vaccination are still limited. Objectives: In this study, we assessed the humoral response to BNT162b2 mRNA COVID-19 vaccine 3 months after the second dose, in a cohort of 495 residents aged $\geq 65$ years from 11 LTCF in Granada, Spain. Method: Between April 19 and April 30, 2021, we measured antiSARS-CoV-2 Spike IgG to evaluate the humoral vaccination response. Antibody titers were reported in binding antibody units $(\mathrm{BAU} / \mathrm{mL})$. Bivariate and multivariate logistic regression models were performed to investigate the impact of age, sex, underlying health conditions, and prior COVID-19 infection on the antibody levels. Results: Over $96 \%$ of the partici-
\end{abstract}

pants developed an adequate humoral response. We detected higher antibody titers in previously infected individuals, compared with those previously uninfected (B: 1,150.059 $\mathrm{BAU} / \mathrm{mL}, p<0.001)$. Moreover, we found a significant inverse association between age and antibody levels $(B:-7.943$ $\mathrm{BAU} / \mathrm{mL}, p<0.05)$. This negative age-dependent response was more noticeable among residents over 85 years old. In contrast, baseline health conditions and cognitive status were not associated with different antibody levels. Conclusions: These findings support monitoring COVID-19 vaccination response trend in older adults, in order to optimize future disease prevention and control strategies in this vulnerable population.

(c) 2021 S. Karger AG, Basel

\section{Introduction}

The COVID-19 pandemic has disproportionately affected older people and particularly those living in longterm care facilities (LTCFs). Residents of LTCFs are at increased risk for COVID-19 infection and severe disease and have been reported to account for $50 \%$ of all COVID- 
19-related deaths in Spain [1]. Therefore, the institutionalized elderly has been considered as a target and prioritized group in COVID-19 vaccination strategies in the EU/EEA [2].

Despite this, actual evidence on vaccine immunogenicity in this vulnerable group is limited, and the duration of humoral protection from vaccination is still unknown. Here, we present early results of post-vaccination immune response at 90 days from a longitudinal cohort study that included 495 residents of 11 LTCFs in the Metropolitan Area of Granada, Andalusia, Spain.

\section{Materials and Methods}

\section{Study Setting and Population}

In Spain, the current vaccination strategy for older adults living in LTCF is based on the administration of 2 doses of the BNT162b2 mRNA (Biontech/Pfizer Comirnaty ${ }^{\mathrm{TM}}$ ) vaccine. The first dose is followed by a second one, at least 21 days later.

The Metropolitan Area of Granada has 55 LTCFs, with approximately 3,000 institutionalised elderly people. For this study, 11 of these 55 LTCFs were randomly selected, and 495 residents aged $\geq 65$ years were subsequently enrolled. Inclusion criteria were age 65 years or older, living in one of the 11 selected LTCFs at the time of vaccination, having received the complete vaccination schedule, and agreeing to participate in the study.

\section{Study Design and Data Collection}

This work is part of the GERO-INMUNO study, a longitudinal multicenter cohort study that started on April 2021. We present the results of the first measurement. To assess the response to the vaccine, we measured anti-Spike IgG levels against severe acute respiratory coronavirus 2 (SARS-CoV-2) among the elderly institutionalized population of the Metropolitan Area of Granada, 3 months (90 days) after the complete BNT162b2 mRNA vaccination schedule. In addition, the association of other individual variables (history of previous COVID-19 infection, comorbidity, cognitive status, sex, and age) on antibody titers was investigated.

Serum samples were analyzed with the DIASORIN LIAISON ${ }^{\circledR}$ SARS-CoV-2 TrimericS IgG test to quantify anti-Spike Glycoprotein (S1) IgG antibodies. Antibody titers were reported in binding antibody units $(\mathrm{BAU} / \mathrm{mL})$, following the World Health Organization International Standards guidelines [3]. According to the manufacturer instructions, the titers were interpreted as positive when they were $\geq 33.8 \mathrm{BAU} / \mathrm{mL}$. The lower and upper limits of detection were $4.8 \mathrm{BAU} / \mathrm{mL}$ and $2,080 \mathrm{BAU} / \mathrm{mL}$, respectively.

All residents included in the study were classified according to history of previous COVID-19 infection, confirmed by polymerase chain reaction, positive IgG serology, or antigen testing. Clinical information on cognitive status, underlying health conditions (diabetes, heart failure, obesity, and chronic obstructive pulmonary disease), and the presence of multimorbidity was also included. Multimorbidity was defined as the coexistence of 2 or more chronic diseases based on a list of long-term conditions usually associated with more complex healthcare needs than those of the gen-

Humoral Response to SARS-CoV-2

Vaccination in Institutionalized Elderly eral population [4]. All clinical and microbiological information was collected from the Digital Patient Medical Records of the Andalusian Public Health System.

\section{Statistical Analyses}

Data were summarized as means \pm standard deviation for continuous variables and as number of patients (\%) for categorical variables. Pearson's $\chi^{2}$ test, Wilcoxon rank-sum test, and KruskalWallis rank-sum test were used, according to the type and distribution of the data, to assess differences in anti-Spike IgG SARS$\mathrm{CoV}-2$ antibody titers between groups. A $p$ value of $<0.05$ was considered statistically significant. Multivariate logistic regression models with 2 -sided $95 \%$ confidence intervals were then constructed to identify independent predictors of the antibody titers. Collinearity of variables was assessed through the variance inflation factor. Analyses were conducted using R Statistical Software (Foundation for Statistical Computing, Vienna, Austria).

\section{Results}

Robust Humoral Response 3 Months Post-BNT162b2

\section{COVID-19 Vaccination}

In our cohort, the first dose of BNT162b2 was administered between December 27, 2021, and January 10, 2021. The second dose was administered between January 18 and 29, 2021. Antibody levels were determined 90 days after receiving the second dose, between April 19 and 30, 2021. The mean age of the 495 residents included in this study was 83.7 years (standard deviation: 8.2 ), and 212 (42.8\%) had confirmed SARS-CoV-2 infection prior to the investigation period.

At 90 days after the second dose of BNT162b2 COVID-19 vaccine, 492 residents developed detectable (titers $\geq 4.8 \mathrm{BAU} / \mathrm{mL}$ ) anti-SARS-CoV-2 Spike IgG antibodies. The humoral response was considered adequate (positive) in 477 (96.1\%) of them, when titers were 33.8 BAU/ $\mathrm{mL}$ or higher, and inadequate (negative) in $18(3.6 \%)$ residents, with titers below $33.8 \mathrm{BAU} / \mathrm{mL}$. None of those classified as negative had confirmed COVID-19 infection prior to vaccination (shown in Table 1).

\section{Impact of Prior Infection and Age on Immunogenicity}

In the bivariate analysis, the mean anti-SARS-CoV-2 Spike IgG antibody levels detected among those with evidence of prior COVID-19 infection were significantly higher than those without prior infection $(p<0.001)$. Multivariate linear regression analysis also showed that prior infectious status correlated significantly with antibody titers $(B: 1,150.059, p<0.001)$. Moreover, a significant inverse relationship was detected between age and antibody levels $(B:-7.943, p<0.05)$ (shown in Table 2 and Fig. 1). 
Table 1. Baseline characteristics of the residents included in the study

\begin{tabular}{|c|c|c|c|c|}
\hline \multirow[t]{2}{*}{ Variables } & \multirow{2}{*}{$\begin{array}{l}\text { Total sample } \\
(n=495)\end{array}$} & \multicolumn{2}{|c|}{ Previous COVID-19 infection } & \multirow[t]{2}{*}{$p$ value } \\
\hline & & yes $(n=212)$ & no $(n=283)$ & \\
\hline \multicolumn{5}{|l|}{ Sex, $n(\%)$} \\
\hline Female & $362(73.5)$ & $154(72.6)$ & $208(73.5)$ & \multirow{2}{*}{$0.831^{a}$} \\
\hline Male & $133(26.5)$ & $58(27.3)$ & $75(26.5)$ & \\
\hline \multicolumn{5}{|l|}{ Age } \\
\hline \multicolumn{5}{|l|}{ Groups, $n(\%)$} \\
\hline $65-75$ years & $87(17.6)$ & $41(19.3)$ & $46(16.2)$ & \multirow{3}{*}{$0.609^{a}$} \\
\hline $76-85$ years & $165(33.3)$ & $67(31.6)$ & $98(34.6)$ & \\
\hline$>85$ years & $243(49.1)$ & $104(49.0)$ & $139(49.1)$ & \\
\hline Continuous, mean (SD) & $83.7(7.9)$ & $83.3(8.0)$ & 83.9 (7.9) & $0.541^{\mathrm{b}}$ \\
\hline \multicolumn{5}{|l|}{ Multimorbidity ${ }^{c}, n(\%)$} \\
\hline Yes & $312(63.6)$ & $134(63.2)$ & $178(62.9)$ & \multirow{2}{*}{$0.943^{a}$} \\
\hline No & $183(36.9)$ & $78(36.8)$ & $105(37.1)$ & \\
\hline \multicolumn{5}{|l|}{ Cognitive impairment, $n$ (\%) } \\
\hline Yes & $171(34.5)$ & $92(43.4)$ & 79 (27.9) & \multirow{2}{*}{$<0.001^{a}$} \\
\hline No & $324(65.4)$ & $120(56.6)$ & $204(72.1)$ & \\
\hline \multicolumn{5}{|l|}{ Heart failure, $n(\%)$} \\
\hline Yes & $44(8.8)$ & $17(8.02)$ & $27(9.5)$ & \multirow{2}{*}{$0.556^{\mathrm{a}}$} \\
\hline No & $451(91.1)$ & 195 (91.9) & $256(90.4)$ & \\
\hline \multicolumn{5}{|c|}{ Type 2 diabetes mellitus, $n(\%)$} \\
\hline Yes & $135(27.2)$ & $48(22.6)$ & $87(30.7)$ & \multirow{2}{*}{$0.045^{\mathrm{a}}$} \\
\hline No & $360(72.2)$ & $164(77.3)$ & $196(69.2)$ & \\
\hline \multicolumn{5}{|l|}{ Obesity (BMI > 30), $n(\%)$} \\
\hline Yes & $52(10.5)$ & $31(14.6)$ & $21(7.42)$ & \multirow{2}{*}{$0.009^{a}$} \\
\hline No & $443(89.4)$ & $181(85.3)$ & $262(92.5)$ & \\
\hline \multicolumn{5}{|c|}{ Chronic obstructive pulmonary disease, $n$ (\%) } \\
\hline Yes & $48(9.7)$ & $20(9.4)$ & $28(9.8)$ & \multirow{2}{*}{$0.864^{\mathrm{a}}$} \\
\hline No & $447(90.3)$ & $192(90.5)$ & $255(90.1)$ & \\
\hline \multicolumn{5}{|l|}{ Antibody response, $n$ (\%) } \\
\hline Yes & $477(96.3)$ & $265(100)$ & $211(93.6)$ & \multirow{2}{*}{$<0.001^{a}$} \\
\hline No & $18(3.6)$ & $0(0)$ & $18(6.4)$ & \\
\hline Antibody titers, mean (SD) & $1,152.1(847.9)$ & $1,811.6(546.3)$ & $658.0(681.2)$ & $<0.001^{b}$ \\
\hline
\end{tabular}

SD, standard deviation. a Pearson's $x^{2}$ test. ${ }^{b}$ Wilcoxon rank-sum test. ${ }^{c}$ Defined as the coexistence of two or more of the following chronic conditions: severe heart disease, autoimmune diseases, chronic kidney disease, respiratory chronic disease, chronic liver disease, inflammatory bowel disease, cerebrovascular accident, or other neurological disease with motor or cognitive deficit, diabetes-related clinical complications, anemia of chronic disorders, incapacitating osteoarticular disease, active neoplasia.

No significant correlation was found between humoral response and sex, comorbidities, multimorbidity, or cognitive impairment $(p>0.05)$. There was no collinearity between the variables included in the model.

\section{Discussion}

We measured the humoral response induced by the BNT162b2 mRNA COVID-19 vaccine in a cohort of 495 institutionalized elderly, in order to gain insight into the immunogenicity of the vaccine, and its durability, 3 months after receiving 2 doses. We observed that more than $96 \%$ of residents presented adequate levels of antiSARS-CoV-2 Spike IgG antibody levels 90 days after the second dose and that titers were considerably higher among those with prior COVID-19 infection.

Several studies have shown an increased humoral response in previously infected individuals, compared to the uninfected ones $[5,6]$, especially in the cases of severe and critical disease [7]. We did not analyze antibody response patterns according to the level of disease severity, but our findings support published evidence suggesting that, also among the elderly and institutionalized, pre- 
Table 2. Antibody titers by sex, age, infectious status, and comorbidities

\begin{tabular}{|c|c|c|c|c|c|c|c|}
\hline Variables & $N$ & Mean & SD & $p$ value $^{\mathrm{a}}$ & $B$ & $95 \% \mathrm{Cl}$ & $\begin{array}{l}\text { Adjusted } \\
p \text { value }^{\mathrm{b}}\end{array}$ \\
\hline \multicolumn{8}{|l|}{ Sex } \\
\hline Male & 133 & $1,211.8$ & 887.5 & 0.425 & -23.315 & -154.0 to 107.3 & 0.724 \\
\hline \multicolumn{8}{|l|}{ Age } \\
\hline \multicolumn{8}{|l|}{ Groups } \\
\hline$>85$ years & 243 & $1,104.6$ & 832.9 & 0.348 & & & \\
\hline Continuous (mean, SD) & & & & & -7.943 & -15.1 to -0.70 & 0.031 \\
\hline \multicolumn{8}{|l|}{ Previous COVID-19 infection } \\
\hline Yes & 212 & $1,811.6$ & 546.4 & \multirow[t]{2}{*}{$<0.001$} & \multirow[t]{2}{*}{$1,150.059$} & \multirow[t]{2}{*}{ 1,035.4-1,264.6 } & \multirow{2}{*}{$<0.001$} \\
\hline No & 283 & 658.1 & 681.2 & & & & \\
\hline \multicolumn{8}{|l|}{ Cognitive impairment } \\
\hline Yes & 171 & $1,287.1$ & 833.9 & \multirow[t]{2}{*}{0.002} & \multirow[t]{2}{*}{8.937} & \multirow[t]{2}{*}{-112.8 to 130.7} & \multirow{2}{*}{0.885} \\
\hline No & 324 & $1,080.9$ & 847.8 & & & & \\
\hline \multicolumn{8}{|l|}{ Heart failure } \\
\hline Yes & 44 & $1,028.0$ & 813.5 & \multirow[t]{2}{*}{0.312} & \multirow[t]{2}{*}{-45.133} & \multirow[t]{2}{*}{-245.3 to 155.1} & \multirow{2}{*}{0.658} \\
\hline No & 451 & $1,164.2$ & 851.0 & & & & \\
\hline \multicolumn{8}{|l|}{ Type 2 diabetes mellitus } \\
\hline Yes & 135 & $1,036.3$ & 847.4 & \multirow[t]{2}{*}{0.067} & -31.005 & -159.7 to 97.7 & \\
\hline No & 360 & $1,195.5$ & 845.1 & & & & 0.636 \\
\hline Obesity (BMI >30) & & & & & & & \\
\hline Yes & 52 & $1,255.3$ & 823.7 & 0.346 & -86.245 & -272.2 to 99.7 & \\
\hline No & 443 & $1,140.0$ & 850.7 & & & & 0.362 \\
\hline Chronic obstructive pulmon & & & & & & & \\
\hline
\end{tabular}

Bivariate and multivariate analysis. SD, standard deviation of the mean; B, unstandardized beta coefficient; $\mathrm{Cl}$, confidence interval. ${ }^{a}$ Bivariate analysis (Wilcoxon and Kruskal-Wallis rank-sum tests). ${ }^{b}$ Multivariate linear regression model adjusted for all the variables shown in the table. Adjusted $R^{2}=0.453$. ${ }^{C}$ Defined as the coexistence of two or more of the following chronic conditions: severe heart disease, autoimmune diseases, chronic kidney disease, respiratory chronic disease, chronic liver disease, inflammatory bowel disease, cerebrovascular accident, or other neurological disease with motor or cognitive deficit, diabetes-related clinical complications, anemia of chronic disorders, incapacitating osteoarticular disease, active neoplasia.

vaccination COVID-19 infection correlates significantly with higher antibody titers $[5,6]$.

Nevertheless, in our cohort, a lower humoral response was detected in older residents. This was more noticeable among residents over 85 years old (shown in Fig. 1). Recent studies also highlighted the negative impact of age on BNT162b2 post-vaccination immunogenicity among LTCFs residents [6]. In line with our findings, this agerelated decline of immunogenicity has been particularly appreciable among individuals aged 80 or more $[7,8]$.

The immunogenicity of some vaccines is known to be suboptimal in the elderly as immunosenescence may neg- atively affect innate and adaptive immune responses and weaken antibody production. The mechanisms by which immunosenescence generates alterations in the immune system, and how these changes impact responses to infection, are still not fully clear; but in older adults, a reduced cellular and humoral response to many different vaccines has been well described. Since the vaccines against influenza, pneumococcal infection, and herpes zoster are specifically recommended and widely used in the elderly, different vaccination strategies have been developed over the last years to improve their immunogenicity (e.g., the use of adjuvants, higher antigen dose, or the administra- 


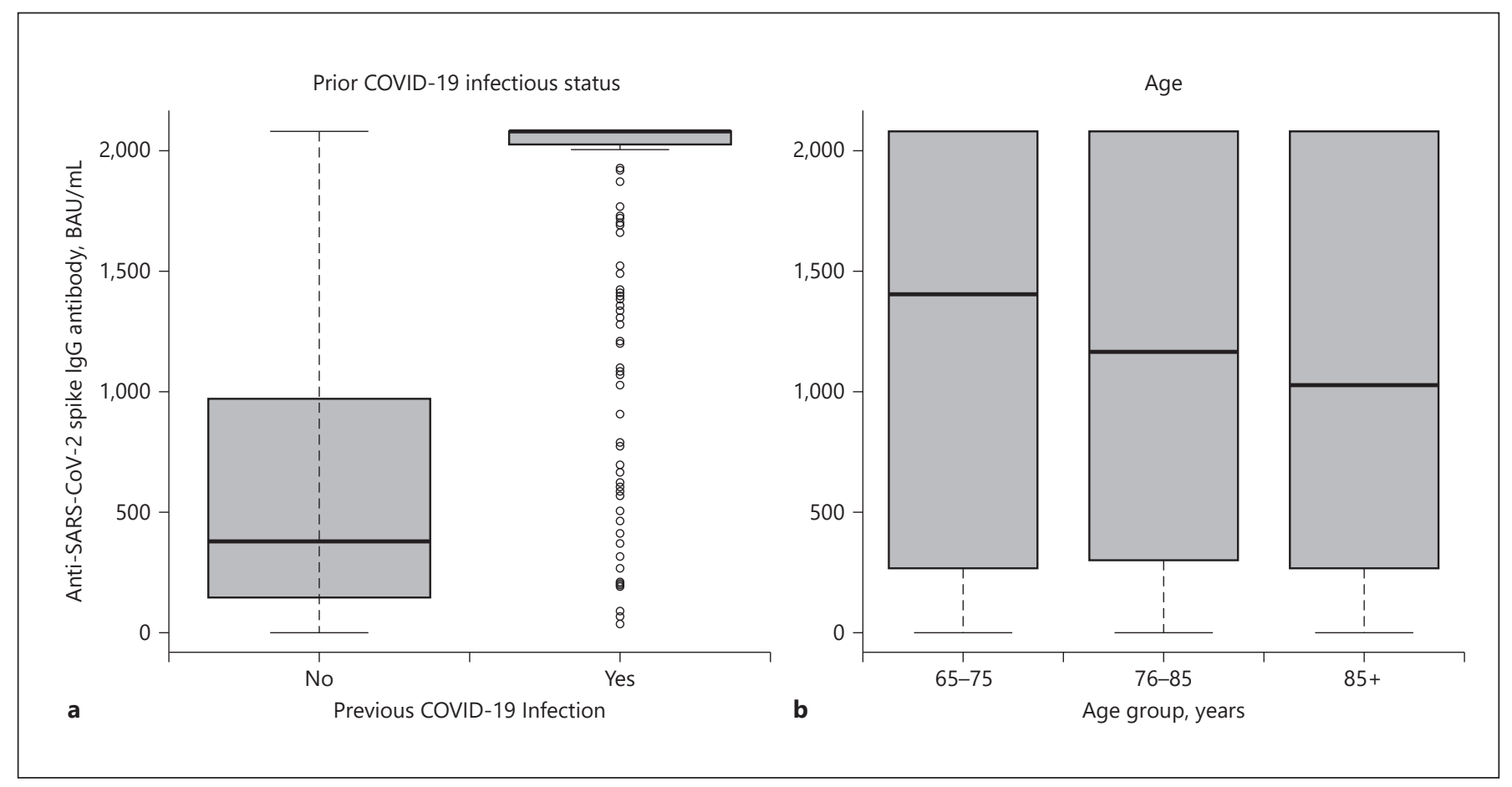

Fig. 1. Anti-SARS-CoV-2 Spike IgG antibody levels, 90 days after second dose of BNT162b2 mRNA COVID-19 vaccine by prior infection status (a) and age (b). The median value is marked by the line inside the box. The interquartile range (IQR) is represented by the lower (25th percentile) and upper (75th percentile) limits of the box. The 10th and 90th percentiles are displayed as horizontal lines outside the box. Outliers are marked as individual points outside the box.

tion of booster shots) $[9,10]$. A recent study suggested that specific strategies to overcome the age-related limitations of COVID-19 vaccination response in the elderly might be necessary in the future [8].

Regarding BNT162b2 mRNA COVID-19 vaccines, there is a growing evidence on their protective effect in residents of LTCFs. Early experiences showed its effectiveness in preventing SARS-CoV-2 infections, hospitalizations, and deaths $[11,12]$. These data are encouraging, but more information are needed on the long-term effects of COVID-19 vaccines in this specific, vulnerable population. Cases of vaccine failure among elderly residents have also been reported. Recent publications have described COVID-19 outbreaks in LTCFs despite high mRNA vaccination rates among residents and staff [1214]. In an outbreak occurred in the north of Italy, with an infection rate of $45 \%$ among fully vaccinated individuals, the only variable associated with an increased risk of infection and death was age [14]. The authors highlighted that integrate vaccination with nonpharmacological measures still remains critical to the control of SARS-CoV-2 transmission in LTCFs setting, especially in the context of new more transmissible variants $[11,13,14]$. Even if viral load in infected vaccinated residents may be lower than that of unvaccinated, the residential environment itself poses some risk factors (closed spaces, specific care needs, proximity between residents and staff) that may facilitate the spread of the virus.

In this study, we reported a good immunogenicity 3 months after vaccination, but our data do not allow us to draw conclusions on the impact of vaccination on disease prevention and control. As described for other vaccines against human viral diseases, investigations on the relationship between vaccine immunogenicity and protection from SARS-CoV-2 infection showed that higher levels of antibody titers significantly correlate with a reduced risk of symptomatic infection $[10,15]$. These data suggest that antibody level may be an important predictor of vaccine efficacy that could help public health response in vulnerable groups $[7,15]$.

In this study, no significant correlation was found between antibody titers and residents' health conditions. Nevertheless, clinical data included in this study may present some limitations since we only considered the 
medical diagnoses available from the official Digital Patient Medical Records. Thus, we were not able to provide a comprehensive geriatric assessment, including other relevant health-related aspects such as functionality and frailty status. Future analyses should consider these variables in order to achieve a broader understanding of these circumstances and their potential implications. Dietary and other lifestyle variables could also add relevant information to future studies. Finally, comparisons with antibody titers in the following months (e.g., 6, 9, or 12 months after second dose) will be necessary to analyze the evolution and duration of the immune response of this population.

Our findings confirm an age-dependent immune response to the vaccine and suggest the need to monitor its evolution in the elderly institutionalized, as a group at increased risk for SARS-CoV-2 infection and severe COVID-19 $[6,11,12]$. Assessing trends in antibody response may support decision-making regarding future disease control and revaccination strategies to ensure adequate protection against infection in this vulnerable population.

\section{Acknowledgments}

The present work is part of the "GERO-INMUNO STUDY," a larger multicenter longitudinal cohort study. We thank Basilio Gómez Pozo for his contribution and methodological advice. We thank all the residents and staff of the facilities included in this study for their cooperation.

\section{Statement of Ethics}

The study was approved by the Research Ethics Committee of Granada (ID 1872-N-20). All procedures performed were in accordance with the 1964 Helsinki Declaration and its later amend- ments or comparable ethical standards. All participants or their legal representative provided written informed consent prior to the inclusion in the study.

\section{Conflict of Interest Statement}

The authors have no conflicts of interest to declare.

\section{Funding Sources}

This study received no specific funding. However, serological tests realized with LIAISON ${ }^{\circledR}$ DIASORIN were covered by DiaSorin S.p.A. The authors declare that DiaSorin S.p.A. has not been involved in any phase of the investigation process; it has not had access to the protocol, nor to the data, and nor to the preliminary or final report.

\section{Author Contributions}

Roberta Causa, Mario Rivera-Izquierdo, and Clara Bermúdez-Tamayo contributed to study conception and design, analyzed the data, and drafted the manuscript. Diego Almagro-Nievas and Begoña López-Hernández contributed to study conception and design. Nicolás Benítez-Muñoz and Maria de la O Soto-Pérez contributed to data collection and management. Fernando García-García and Marta Alvarez-Estévez were responsible for the laboratory analyses. All authors contributed to data interpretation, revised, and approved the final version of the manuscript.

\section{Data Availability Statement}

A full, anonymized version of the data that support the findings of this study is available from the corresponding author upon reasonable request. The data are not publicly available due to ethical restrictions, to guarantee the privacy of research participants.

\section{References}

1 European Centre for Disease Prevention and Control (ECDC). Surveillance data from public online national reports on COVID-19 in longterm care facilities. Stockholm: ECDC; 2021 Mar 31 [cited 2021 Jun 26]. Available from: https://www.ecdc.europa.eu/en/all-topics-z/ coronavirus/threats-and-outbreaks/covid-19/ prevention-and-control/LTCF-data.

2 European Centre for Disease Prevention and Control. COVID-19 vaccination and prioritisation strategies in the EU/EEA. Stockholm: ECDC; 2020 Dec 22 [cited 2021 Jun 26]. Available from: https://www.ecdc.europa.eu/ en/publications-data/covid-19-vaccinationand-prioritisation-strategies-eueea.
3 Kristiansen PA, Page M, Bernasconi V, Mattiuzzo G, Dull P, Makar K, et al. WHO International Standard for anti-SARS-CoV-2 immunoglobulin. Lancet. 2021;397(10282):1347-8.

4 Johnston MC, Crilly M, Black C, Prescott GJ, Mercer SW. Defining and measuring multimorbidity: a systematic review of systematic reviews. Eur J Public Health. 2019;29(1):182-9.

5 Salmerón Ríos S, Mas Romero M, Cortés Zamora EB, Tabernero Sahuquillo MT, Romero Rizos L, Sánchez-Jurado PM, et al. Immunogenicity of the BNT162b2 vaccine in frail or disabled nursing home residents: $\mathrm{CO}-$ VID-A study. J Am Geriatr Soc. 2021;69(6): 1441-7.
6 Canaday DH, Carias L, Oyebanji OA, Keresztesy D, Wilk D, Payne M, et al. Reduced BNT162b2 mRNA vaccine response in SARSCoV-2-naive nursing home residents. Clin Infect Dis. 2021. ciab447.

7 Collier DA, Ferreira IATM, Kotagiri P, Datir RP, Lim EY, Touizer E, et al. Age-related immune response heterogeneity to SARS-CoV-2 vaccine BNT162b2. Nature. 2021;596(7872): $417-22$.

8 Müller L, Andrée M, Moskorz W, Drexler I, Walotka L, Grothmann R, et al. Age-dependent immune response to the Biontech/Pfizer BNT162b2 COVID-19 vaccination. Clin Infect Dis. 2021. ciab381.
Humoral Response to SARS-CoV-2 Vaccination in Institutionalized Elderly 
9 Gustafson CE, Kim C, Weyand CM, Goronzy $\mathrm{JJ}$. Influence of immune aging on vaccine responses. J Allergy Clin Immunol. 2020; 145(5):1309-21.

10 Neurath AR. Immune response to viruses: antibody-mediated immunity. Encycl Virol. 2008:56-70.

11 Shrotri M, Krutikov M, Palmer T, Giddings R, Azmi B, Subbarao S, et al. Vaccine effectiveness of the first dose of ChAdOx1 nCoV-19 and BNT162b2 against SARS-CoV-2 infection in residents of long-term care facilities in England (VIVALDI): a prospective cohort study. Lancet Infect Dis. 2021;23(21):S147300289-30999.
12 Mazagatos C, Monge S, Olmedo C, Vega L, Gallego P, Martín-Merino E, et al. Effectiveness of mRNA COVID-19 vaccines in preventing SARS-CoV-2 infections and COVID-19 hospitalisations and deaths in elderly long-term care facility residents, Spain, weeks 532020 to 13 2021. Euro Surveill. 2021; 26(24):2100452.

13 Williams C, Al-Bargash D, Macalintal C, Stuart R, Seth A, Latham J, et al. COVID-19 outbreak associated with a SARS-CoV-2 P.1 lineage in a long-term care home after implementation of a vaccination program: Ontario, April-May 2021. Clin Infect Dis. 2021. ciab617.
14 Faggiano F, Rossi MA, Cena T, Milano F, Barale A, Ristagno Q, et al. An outbreak of COVID-19 among mRNA-vaccinated nursing home residents. Vaccines. 2021;9(8):859.

15 Khoury DS, Cromer D, Reynaldi A, Schlub TE, Wheatley AK, Juno JA, et al. Neutralizing antibody levels are highly predictive of immune protection from symptomatic SARSCoV-2 infection. Nat Med. 2021;27(7):120511. 